\title{
HIV AND ART-RELATED FAT ALTERATIONS
}

nature reviews disease primers
In some patients, HIV infection and antiretroviral therapy (ART) are associated with alterations in fat, including subcutaneous fat loss (lipoatrophy) and/or abdominal fat gain (lipohypertrophy) or generalized fat gain.

\section{EPIDEMIOLOGY}

Lipoatrophy was first identified in people living with HIV (PLWH) who received first-generation thymidine analogue nucleoside reverse transcriptase inhibitors (NRTIs). Up to $70 \%$ of PLWH who receive ART have abdominal or visceral fat gain. Weight gain and generalized fat gain occur in almost all PLWH following initiation of ART, although some integrase strand transfer inhibitors are associated with a higher fat gain than other classes of ART; this is also the case for tenofovir alafenamide. Ectopic fat deposits in the liver, heart
and skeletal muscle have been reported in PLWH who receive combination ART

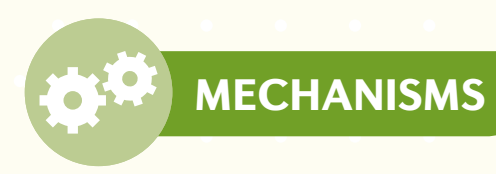

Viral proteins -

such as Vpr, Nef and

Tat - are released

and exert effects

on adjacent cells,

such as altered

expression of genes

that have a role in

I lipid accumulation,

mitochondrial

dysfunction and

fibrosis

ADIPOCYTE —
Tat - are released
- - - - - - - - - - -

Alterations in the

adipose immune

system - such

as increased

proinflammatory

cytokine productio

and an increased

number of $\mathrm{CD}^{+}$

T cells - have bee

: observed in PLWH

\section{PREVENTION}

Lipoatrophy can be prevented by avoiding first-generation ARTs tha carry a high risk of this condition, such as zidovudine and stavudine.
Close monitoring of weight gain, together with lifestyle intervention such as healthy diet and regular exercise, can help to prevent HIV and ART-associated lipohypertrophy and generalized fat gain.
Some ARTs can also affect adipose function through altered
thos adipogenesis, mitochondrial dysfunction and fibrosis
For the Primer, visit doi:10.1038/541572-020-0190-0

\section{MANAGEMENT}

Facial lipoatrophy can be partially reversed by switching medication. However, plastic surgery (commonly the injection of poly-L-lactic acid into the cheek) can be used in those with permanent lipoatrophy. Medication switching is generally not effective to reduce abdominal obesity; patients should adopt a reduced-calorie diet and a regular exercise programme. Pharmacological therapy using tesamorelin is approved for the treatment of abdominal obesity in PLWH in the US and Canada, among other countries.

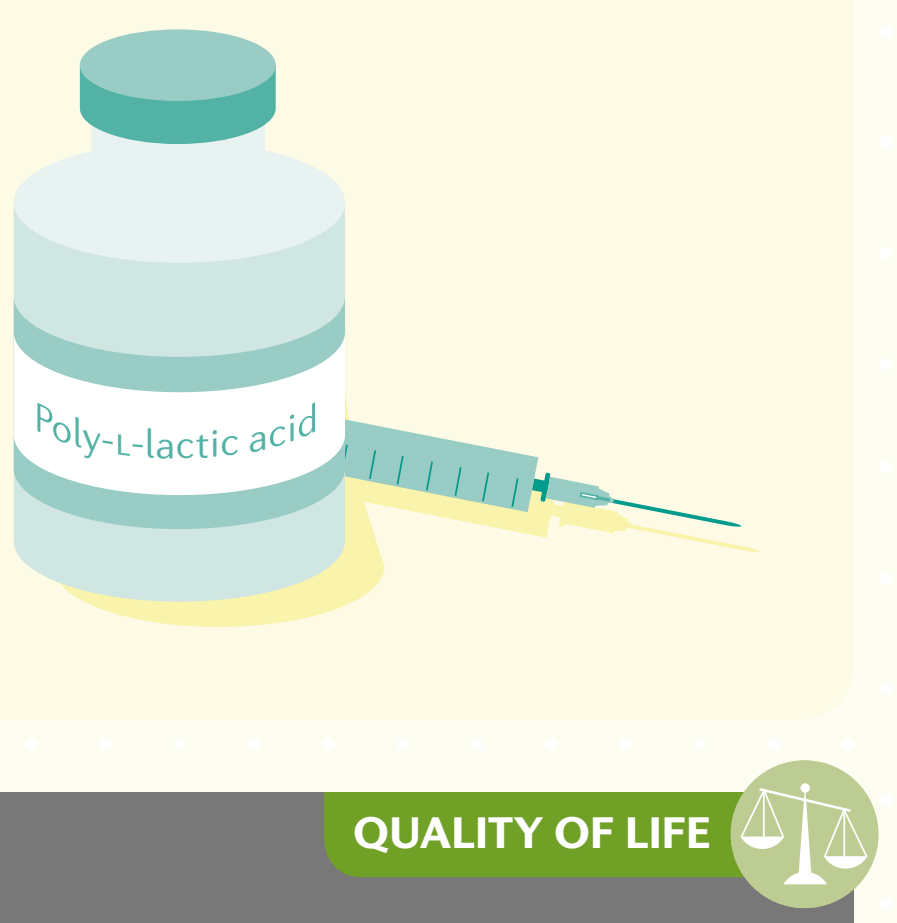

Research assessing the effect of fat alterations on health-related quality of life in PLWH is scarce. Some quality of life instruments include questions specifically about fat alterations, such as the Assessment of Body Change and Distress and the HIV/AIDS-targeted quality of life tool. Importantly, the use of facial plastic surgery to treat facial lipoatrophy has been associated with improved quality of life scores in PLWH. 